\title{
Type Reduction Operators for Interval Type-2 Defuzzification
}

\author{
Thomas A. Runkler ${ }^{\mathrm{a}}$, Chao Chen ${ }^{\mathrm{b}}$, Robert John ${ }^{\mathrm{c}}$ \\ ${ }^{a}$ Siemens AG, Corporate Technology, 81730 Munich, Germany, Email: \\ Thomas.Runkler@siemens.com \\ ${ }^{b}$ Laboratory for Uncertainty in Data and Decision Making (LUCID), University of \\ Nottingham, Wollaton Road, Nottingham, NG8 1BB, UK \\ Email:Chao.Chen@nottingham.ac.uk \\ ${ }^{c}$ Laboratory for Uncertainty in Data and Decision Making (LUCID), University of \\ Nottingham, Wollaton Road, Nottingham, NG8 1BB, UK \\ Email: Robert.John@nottingham.ac.uk
}

\begin{abstract}
Fuzzy sets are an important approach to model uncertainty. Defuzzification maps fuzzy sets to non-fuzzy (crisp) values. Type-2 fuzzy sets model uncertainty in the degree of membership in a fuzzy set. Type- 2 defuzzification maps type-2 fuzzy sets to non-fuzzy values. Type reduction maps type- 2 fuzzy sets to type -1 fuzzy sets, in order to make type- 2 defuzzification easier and to implement more efficient type- 2 defuzzification algorithms.

This paper is a first step towards a theoretical foundation of the emerging field of type reduction. Five mathematical properties of type reduction are defined, and two existing type reduction methods (Nie-Tan and uncertainty weight) are examined with respect to our five properties. Furthermore, two new type reduction methods are proposed: consistent linear type reduction and consistent quadratic type reduction. All our five properties are satisfied by consistent quadratic type reduction.
\end{abstract}

Keywords: defuzzification, type-2 fuzzy sets

This manuscript is the authors' original work and has not been published nor has it been submitted simultaneously elsewhere. All authors have checked the manuscript and have agreed to the submission.

Preprint submitted to Information Sciences

August 6, 2018 


\section{Introduction}

Fuzzy sets [29] are an important approach to model and process uncertain information. Fuzzy information processing (for example in fuzzy rule based systems $[14,26])$ usually yield fuzzy sets as outputs. If non-fuzzy outputs are needed (for example in control applications $[7,18]$ ), these can be obtained from fuzzy outputs using defuzzification. Many defuzzification methods have been proposed during the last decades, for an overview see [19, 20, 25], and the mathematical properties of defuzzification have been studied in [24].

Interval type-2 fuzzy sets $[13,15,30]$ are extensions of (type-1) fuzzy sets, where the degrees of membership in a fuzzy set may be uncertain. Interval type-2 fuzzy information processing yields interval type- 2 fuzzy sets as outputs, which can be converted to non-fuzzy outputs using interval type- 2 defuzzification. One of the most popular methods for interval type- 2 defuzzification is the Karnik-Mendel algorithm [11]. The mathematical properties of interval type-2 defuzzification have been studied in [22].

Recently, alternative methods for interval type -2 defuzzification have been proposed that are based on type reduction. Type reduction of an interval type- 2 fuzzy set means a mapping to a type- 1 fuzzy set which then can be defuzzified using type-1 defuzzification techniques. Examples are the Nie-Tan (NT) [17] and the uncertainty weight (UW) method [23]. The relation between type1 defuzzification, type- 2 defuzzification, and type reduction is illustrated in Fig. 1.

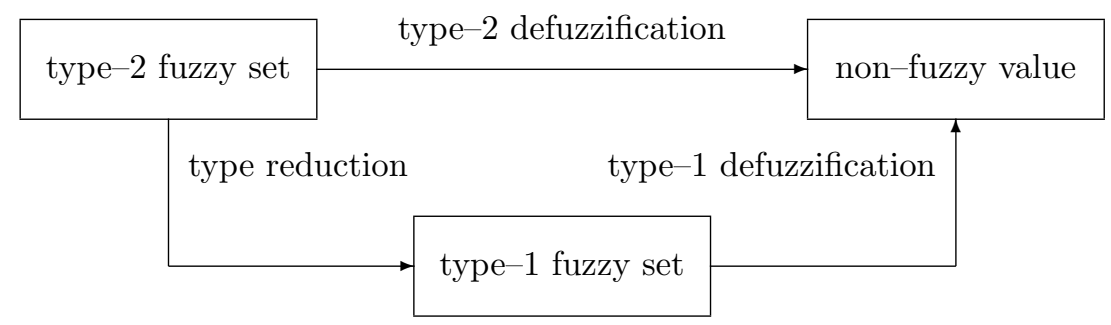

Figure 1: Type-1 and type-2 defuzzification and type reduction. 
The NT and UW methods (and also the CQTR and CLTR methods proposed in this paper) are different from the Karnik-Mendel approach.These methods reduce each interval type- 2 fuzzy set to a type- 1 fuzzy set upfront, and then perform ordinary type -1 defuzzification. These methods can therefore also be viewed as a tool to design type-1 membership functions in scenarios with different levels of uncertainty for different decision options.

Type-reduction based interval type-2 defuzzification reduces interval type2 fuzzy sets to type -1 fuzzy sets upfront, so we lose some ability to explicitly consider the specific uncertainty of the memberships which is considered an important advantage of interval type -2 fuzzy sets over type -1 fuzzy sets. It was shown however for typical fuzzy controller scenarios that the NT method and its variants yield very good approximations of the Karnik-Mendel algorithm at a reduced computational cost $[12,23]$, which appears beneficial for a large number of real-world applications.

The emerging field of type reduction needs a theoretical foundation that enables researchers to assess, compare, and develop novel type reduction methods. So the focus of this paper is not just proposing new type reduction formulas. We also propose the required properties (a standard) for designing type reduction methods. As a first step in this direction, this paper defines five mathematical properties of type reduction. There are infinitely many choices for type reduction functions. An important criterion for type reduction functions is (mathematical and computational) simplicity, so we will focus on linear and quadratic functions in this paper. We will derive specific linear and quadratic functions to meet our five proposed properties, which will lead us to propose two new type reduction methods, consistent linear type reduction (CLTR) and consistent quadratic type reduction (CQTR).

We start with a brief review of type- 1 and interval type- 2 defuzzification, including the NT and UW methods (Section 2). Then we define five mathematical properties for type reduction (Section 3) and evaluate the properties of the NT and UW methods (Sections 4 and 5). Further, we propose the new CLTR and CQTR type reduction methods and discuss their properties (Sections 
6 and 7). Finally, we summarize the properties of all considered methods, give some illustrative examples (Section 8), and present our conclusions and ideas for future research (Section 9).

\section{Type-1 and Type-2 Defuzzification}

A type-1 fuzzy set [29] over a universe $X$ is specified by a membership function $u: X \rightarrow[0,1]$, so $u(x)$ quantifies the degree of membership of any $x \in X$ in the type -1 fuzzy set. Type -1 defuzzification is a function $d$ that maps any type-1 fuzzy membership function to one representative crisp (non-fuzzy) value in $X$.

$$
d(u(x)) \in X
$$

One of the most popular type- 1 defuzzification methods is the centroid defined as

$$
d_{\mathrm{C}}(u(x))=\frac{\int_{X} u(x) \cdot x d x}{\int_{X} u(x) d x}
$$

which represents the (horizontal) position of the center of gravity (first moment) of the area between the membership function and the $x$ coordinate (abscissa). Many other methods for type -1 defuzzification have been proposed in the literature. For an overview see $[19,20,25]$.

An interval-fuzzy set $[3,9,10]$ or closed interval type -2 fuzzy set $[16]$ is specified by two membership functions: a lower membership function $\underline{u}: X \rightarrow$ $[0,1]$ and an upper membership function $\bar{u}: X \rightarrow[0,1]$, where

$$
\underline{u}(x) \leq \bar{u}(x)
$$

for all $x \in X$. Interval type -2 fuzzy sets can be used to model uncertainty about a membership function or to model risk in decision processes [21].

A popular interval type-2 defuzzification method is the Karnik-Mendel (KM) algorithm [11] that considers all (type-1) membership functions $u(x)$ between the lower and upper membership functions $\underline{u}(x)$ and $\bar{u}(x)$, respectively,

$$
\underline{u}(x) \leq u(x) \leq \bar{u}(x)
$$


For one dimensional universes $X \subseteq \mathbb{R}$, KM computes the lower and upper centroids that can be written as

$$
\begin{aligned}
& \underline{d}_{C}(\underline{u}(x), \bar{u}(x))=\inf _{\underline{u}(x) \leq u(x) \leq \bar{u}(x)} d_{\mathrm{C}}(u(x)) \\
& \bar{d}_{C}(\underline{u}(x), \bar{u}(x))=\sup _{\underline{u}(x) \leq u(x) \leq \bar{u}(x)} d_{\mathrm{C}}(u(x))
\end{aligned}
$$

and returns the average of these

$$
d_{\mathrm{KM}}(\underline{u}(x), \bar{u}(x))=\frac{\underline{d}_{C}(\underline{u}(x), \bar{u}(x))+\bar{d}_{C}(\underline{u}(x), \bar{u}(x))}{2}
$$

The Karnik-Mendel algorithm is iterative and computationally intensive, so several enhanced methods and efficient implementations have been proposed in the literature $[6,27,28]$.

Nie and Tan [17] have introduced an alternative way to interval type-2 defuzzification. The idea is to transform the lower and upper membership functions $\underline{u}(x)$ and $\bar{u}(x)$ to one single (type-1) membership function $u(x)$, and then apply type -1 defuzzification, for example the centroid method, to $u(x)$. The conversion of the lower and upper interval type -2 membership functions to one single type -1 membership function is called type reduction. Type reduction for interval type- 2 membership functions can be viewed as an application of a twodimensional fusion function $[1,4]$. In their original paper, Nie and Tan [17] proposed the Nie-Tan (NT) type reduction formula

$$
u_{\mathrm{NT}}(x)=\frac{u(x)+\bar{u}(x)}{2}
$$

which converts an interval type- 2 fuzzy set to a type -1 fuzzy set by taking the average of each respective upper and lower membership grades.

Recently, Runkler et al. [23] presented an alternative type reduction formula called the uncertainty weight $(U W)$ method

$$
u_{\mathrm{UW}}(x)=\frac{1}{2}(\underline{u}(x)+\bar{u}(x)) \cdot(1+\underline{u}(x)-\bar{u}(x))^{\alpha}
$$

with a parameter $\alpha>0$ that quantifies to which degree uncertainty is taken into account in defuzzification. Rather than simply taking the average of each 
respective upper and lower membership grades (which is used in the Nie-Tan method), the uncertainty weight method multiplies this average by the weight factor $(1+\underline{u}(x)-\bar{u}(x))^{\alpha}$, which is zero for maximum uncertainty $(\underline{u}(x)=0$, $\bar{u}(x)=1)$ and one for minimum uncertainty $(\underline{u}(x)=\bar{u}(x))$, and which increases linearly with the uncertainty for $\alpha=1$, less than linearly for all $\alpha<1$ and more than linearly for all $\alpha>1$. The experiments in $[12,23]$ indicated that both NT and UW can be used to yield good approximations of KM with low computational effort. Fig. 2 shows the defuzzification results of the KM, NT, and UW $(\alpha=1)$ methods for an example of a triangular interval type- 2 fuzzy set. The vertical solid lines indicate the defuzzification results for the three methods: $d_{\mathrm{KM}}=0.4650, d_{\mathrm{NT}}=0.4667$, and $d_{\mathrm{UW}}=0.4727$, which are all very similar for this example. The dashed curves at the NT and UW graphs are the results of type reduction. The KM graph does not show any dashed curve because KM directly produces a non-fuzzy value from a type-2 fuzzy set, without type reduction to a type- 1 fuzzy set. NT type reduction yields a (type-1) membership function that passes right through the middle of the grey area limited by the upper and lower interval type -2 membership functions. In this example, UW type reduction yields a membership function partially lying outside the grey area. The observation of this behavior was one of the main motivations to define the properties of type reduction (most specifically the so-called type- 2 consistency property) which are introduced in the following section.

\section{Properties of Type Reduction}

This section follows a similar approach as early work on properties of type-1 defuzzification operators [24] and their extension to interval type-2 defuzzification operators [22]. Based on intuitive requirements we define five mathematical properties of type reduction methods for interval type- 2 defuzzification and examine relations between these properties. This list of properties is not intended to be complete, but should serve as a basis for discussion and extension. The 

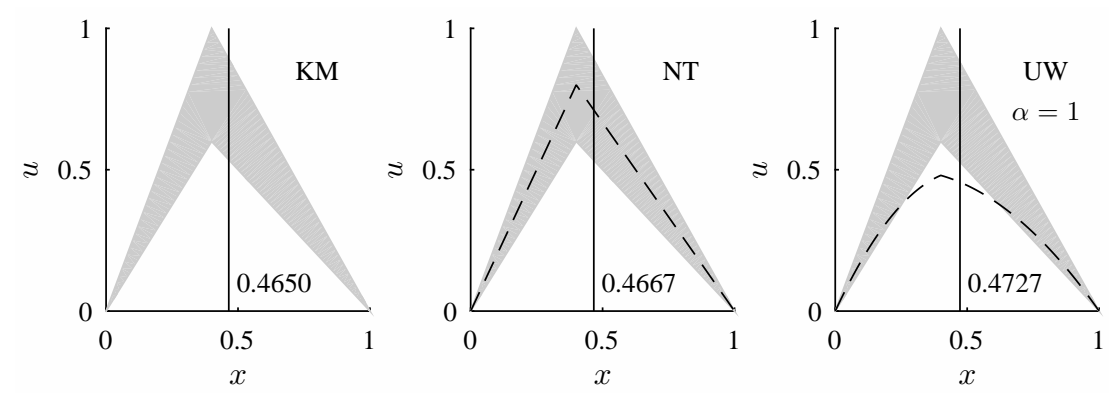

Figure 2: Defuzzification of a triangular interval type-2 fuzzy set using the KM, NT, and UW $(\alpha=1)$ methods.

purpose of these properties is to evaluate, compare, and develop type reduction methods.

Here, we only consider pointwise type reduction methods, i.e. for the computation of a type- 1 membership degree $u(x)$ we only consider the lower and upper interval type-2 membership degrees $\underline{u}(x)$ and $\bar{u}(x)$ at the same point $x$, but not the membership degree $\underline{u}(y)$ and $\bar{u}(y)$ at any other point $y \neq x$. Therefore, we can omit the argument $x$ and simply write each type reduction method as a function $f:[0,1]^{2} \rightarrow[0,1]$ with

$$
u=f(\underline{u}, \bar{u})
$$

So we will always consider the type -1 and type -2 memberships of only one single element at a time. If we want to use the function $f$ to reduce the lower and upper interval type- 2 membership functions $\underline{u}(x)$ and $\bar{u}(x)$ to a type- 1 membership function $u(x)$, then we have to apply the function $f$ to each $x \in X$. The graph of each type reduction function $f$ can be visualized in a three-dimensional diagram with the coordinate axes $\underline{u}, \bar{u}$, and $u$. We will show such diagrams for each of the discussed type reduction functions in the corresponding Sections 4-7. 


\subsection{Type-1 Consistency}

If the lower and upper interval type-2 memberships are equal, then we obtain the special case of type -1 memberships.

$$
\underline{u}=\bar{u}
$$

In this case there no uncertainty about the membership, and we expect the type reduction to yield

$$
\Rightarrow \quad u=\underline{u}=\bar{u}
$$

This means that if the uncertainty disappears, the interval type-2 fuzzy model will reduce to a conventional type -1 fuzzy model. This property corresponds to the (only) requirement that was used to derive the popular Karnik-Mendel method. It also corresponds to the notion of idempotency, which is very well known in algebra and in the field of aggregation functions [2].

Definition 1. A type reduction function $f$ is type-1 consistent if and only if

$$
f(u, u)=u \text { for all } u \in[0,1]
$$

\subsection{Type-2 Consistency}

Let us assume arbitrary lower and upper interval type -2 memberships $\underline{u}, \bar{u} \in$ $[0,1]$ with

$$
\underline{u} \leq \bar{u}
$$

A common interpretation of this situation is that there is uncertainty about the membership, and the membership is at least $\underline{u}$ and at most $\bar{u}$.

$$
\underline{u} \leq u \leq \bar{u}
$$

This means that the effective (type-1) memberships produced by type reduction will never be outside the interval of uncertainty specified for the type-2 memberships, never lower than the lower bound, and never higher that the upper bound. 
Definition 2. A type reduction function $f$ is type-2 consistent if and only if

$$
\underline{u} \leq f(\underline{u}, \bar{u}) \leq \bar{u} \text { for all } \underline{u}, \bar{u} \in[0,1] \text { with } \underline{u} \leq \bar{u}
$$

Theorem 1. If a function is type-2 consistent, then it is type-1 consistent.

Proof. Trivial.

Type- 2 consistency is related to the notion of an averaging function (a function which is between the minimum and the maximum), but it is not the same because we are not assuming symmetry here. Type reduction is also closely related to the idea of ignorance functions and entropy for interval-valued fuzzy sets [5]. We are planning to investigate in a future project if adding a symmetry constraint could yield a generalized definition of type reduction in the context of averaging functions, and we want to have a closer look at the relation between type reduction and ignorance functions.

\section{3. (Strict) Uncertainty Conformity}

Let us assume that the membership is around $u^{*} \in[0,1]$ with an uncertainty of $\pm \Delta u$, where

$$
u^{*}-\Delta u \geq 0, \quad u^{*}+\Delta u \leq 1
$$

so we obtain

$$
\underline{u}=u^{*}-\Delta u, \quad \bar{u}=u^{*}+\Delta u
$$

For $\Delta u=0$ we have the type -1 case, and for any type -1 consistent function $f$ we obtain

$$
u=f\left(u^{*}, u^{*}\right)=u^{*}
$$

If we increase $\Delta u$, then the average membership $u^{*}$ stays the same, but the uncertainty around $u^{*}$ increases. If we have two objects $x_{1}, x_{2} \in X$ both with memberships around $u^{*}$, one with uncertainty $\Delta u_{1}$ and the other with a higher uncertainty $\Delta u_{2}, \Delta u_{1} \leq \Delta u_{2}$, then we may want to give the first (i.e. more certain) object $x_{1}$ a larger weight than $x_{2}, u\left(x_{1}\right)>u\left(x_{2}\right)$. This leads us to require that $u$ should be (strictly) monotonically decreasing with $\Delta u$. If a 
type reduction operator has this property, then for two options with the same average membership but with different levels of uncertainty (spread between the lower and upper type-2 memberships) the option with lower uncertainty will be preferred over the option with higher uncertainty, so the degree of uncertainty is reflected in the type reduction process.

Definition 3. A type reduction function $f$ is uncertainty conform if and only if for any $u^{*} \in[0,1]$ and any $\Delta u_{1}, \Delta u_{2} \geq 0, \Delta u_{1}<\Delta u_{2}$ with

$$
u^{*}-\Delta u_{2} \geq 0, \quad u^{*}+\Delta u_{2} \leq 1
$$

the following condition holds:

$$
f\left(u^{*}-\Delta u_{1}, u^{*}+\Delta u_{1}\right) \geq f\left(u^{*}-\Delta u_{2}, u^{*}+\Delta u_{2}\right)
$$

Definition 4. A type reduction function $f$ is strictly uncertainty conform if and only if for any $u^{*} \in[0,1]$ and any $\Delta u_{1}, \Delta u_{2} \geq 0, \Delta u_{1}<\Delta u_{2}$ with

$$
u^{*}-\Delta u_{2} \geq 0, \quad u^{*}+\Delta u_{2} \leq 1
$$

the following condition holds:

$$
f\left(u^{*}-\Delta u_{1}, u^{*}+\Delta u_{1}\right)>f\left(u^{*}-\Delta u_{2}, u^{*}+\Delta u_{2}\right)
$$

Lemma 1. The preconditions of Definitions 3 and 4 imply $\Delta u \leq 0.5$.

Proof.

$$
\begin{array}{ll}
\text { For } u^{*} \in[0,0.5]: & u^{*}-\Delta u \geq 0 \Rightarrow \Delta u \leq u^{*} \leq 0.5 \\
\text { For } u^{*} \in[0.5,1]: & u^{*}+\Delta u \leq 1 \Rightarrow \Delta u \leq 1-u^{*} \leq 0.5
\end{array}
$$

So for $u^{*} \in[0,1]$ we have $\Delta u \leq 0.5$.

Theorem 2. If a function is strictly uncertainty conform, then it is uncertainty conform.

Proof. Trivial. 


\subsection{Ignorance of Indifference}

The most uncertain interval type- 2 membership values are

$$
\underline{u}=0, \quad \bar{u}=1
$$

In this case we have no information about the membership at all, so we may want to completely ignore such indifferent objects

$$
\Rightarrow \quad u=0
$$

If a type reduction operator has this property, then options with complete uncertainty will receive zero memberships and hence will receive weight zero in the defuzzification process.

Definition 5. A type reduction function $f$ ignores indifference if and only if

$$
f(0,1)=0
$$

\section{Properties of the Nie-Tan Method}

The Nie-Tan (NT) method [17] is specified by the type reduction function

$$
f_{\mathrm{NT}}(\underline{u}, \bar{u})=\frac{\underline{u}+\bar{u}}{2}
$$

In this section we examine $f_{\mathrm{NT}}$ with respect to the five properties defined in the previous section. The properties of the NT and all other methods considered in this paper will be summarized in section 8 .

Theorem 3. The Nie-Tan type reduction function is type-1 consistent.

Proof.

$$
f_{\mathrm{NT}}(u, u)=\frac{u+u}{2}=u \text { for all } u \in[0,1]
$$

Theorem 4. The Nie-Tan type reduction function is type-2 consistent.

Proof. For all $\underline{u}, \bar{u} \in[0,1]$ with $\underline{u} \leq \bar{u}$

$$
\begin{aligned}
& f_{\mathrm{NT}}(\underline{u}, \bar{u})=\frac{\underline{u}+\bar{u}}{2} \geq \frac{\underline{u}+\underline{u}}{2}=\underline{u} \\
& f_{\mathrm{NT}}(\underline{u}, \bar{u})=\frac{\underline{u}+\bar{u}}{2} \leq \frac{\bar{u}+\bar{u}}{2}=\bar{u}
\end{aligned}
$$


Theorem 5. The Nie-Tan type reduction function is uncertainty conform.

Proof. For any $u^{*} \in[0,1]$ and any $\Delta u \geq 0$ we obtain

$$
f_{\mathrm{NT}}\left(u^{*}-\Delta u, u^{*}+\Delta u\right)=\frac{u^{*}-\Delta u+u^{*}+\Delta u}{2}=u^{*}
$$

so for $\Delta u_{1} \geq \Delta u_{2}$

$$
\begin{aligned}
f_{\mathrm{NT}}\left(u^{*}-\Delta u_{1}, u^{*}+\Delta u_{1}\right) & =f_{\mathrm{NT}}\left(u^{*}-\Delta u_{2}, u^{*}+\Delta u_{2}\right)=u^{*}(34) \\
\Rightarrow \quad f_{\mathrm{NT}}\left(u^{*}-\Delta u_{1}, u^{*}+\Delta u_{1}\right) & \geq f_{\mathrm{NT}}\left(u^{*}-\Delta u_{2}, u^{*}+\Delta u_{2}\right)
\end{aligned}
$$

Theorem 6. The Nie-Tan type reduction function is not strictly uncertainty conform.

Proof. From (34) follows

$$
f_{\mathrm{NT}}\left(u^{*}-\Delta u_{1}, u^{*}+\Delta u_{1}\right) \ngtr f_{\mathrm{NT}}\left(u^{*}-\Delta u_{2}, u^{*}+\Delta u_{2}\right)
$$

Theorem 7. The Nie-Tan type reduction function does not ignore indifference.

Proof.

$$
f_{\mathrm{NT}}(0,1)=\frac{0+1}{2}=0.5 \neq 0
$$

Fig. 3 shows the graph of the NT function over the triangle $(\underline{u}, \bar{u}) \in[0,1]^{2}$ with $\underline{u} \leq \bar{u}$. The graph of the NT function is a triangle with the edge points $(\underline{u}, \bar{u}, u)=$ $(0,0,0),(1,1,1)$, and $(0,1,0.5)$, which contradicts ignorance of indifference. The graph of the NT function is linear with a slope of 0.5 along both the $\underline{u}$ and $\bar{u}$ axes.

\section{Properties of the Uncertainty Weight Method}

Runkler et al. proposed the uncertainty weight (UW) method [23] specified by the type reduction function

$$
f_{\mathrm{UW}}(\underline{u}, \bar{u})=\frac{1}{2}(\underline{u}+\bar{u}) \cdot(1+\underline{u}-\bar{u})^{\alpha}
$$

with the parameter $\alpha>0$. Obviously, UW converges to NT as $\alpha$ approaches 0 .

$$
\lim _{\alpha \rightarrow 0} f_{\mathrm{UW}}=f_{\mathrm{NT}}
$$




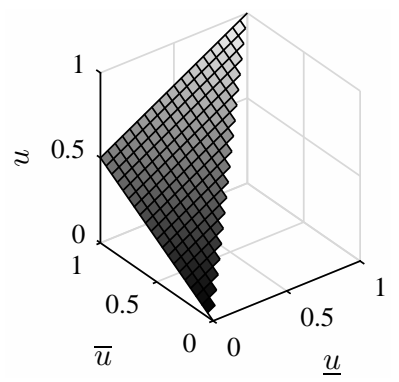

Figure 3: Graph of the NT function.

In this section we examine $f_{\mathrm{UW}}$ with respect to the five properties defined in this paper.

Theorem 8. The uncertainty weight type reduction function is type-1 consistent.

Proof.

$$
f_{\mathrm{UW}}(u, u)=\frac{1}{2}(u+u) \cdot(1+u-u)^{\alpha}=u \text { for all } u \in[0,1]
$$

Theorem 9. The uncertainty weight type reduction function is not generally (i.e. for any $\alpha>0$ ) type-2 consistent.

Proof. Let for example $\underline{u}=0.5, \bar{u}=1$. Then

$$
\begin{gathered}
f_{\mathrm{UW}}(0.5,1)=\frac{1}{2}(0.5+1) \cdot(1+0.5-1)^{\alpha}=0.5 \frac{3}{2^{\alpha+1}} \geq 0.5=\underline{u} \\
\text { for } 2^{\alpha+1}>3 \Rightarrow \alpha>\frac{\log 3}{\log 2}-1 \approx 0.585
\end{gathered}
$$

Theorem 10. The uncertainty weight type reduction function is strictly uncertainty conform.

Proof.

$$
\begin{gathered}
f_{\mathrm{UW}}\left(u^{*}-\Delta u, u^{*}+\Delta u\right) \\
=\frac{1}{2}\left(u^{*}-\Delta u+u^{*}+\Delta u\right) \cdot\left(1+u^{*}-\Delta u-u^{*}-\Delta u\right)^{\alpha}=u^{*}(1-2 \Delta u)^{\alpha}
\end{gathered}
$$

is strictly monotocially decreasing with $\Delta u$ for $\Delta u \leq 0.5$ (Lemma 1) and for $\alpha>0$. 
Theorem 11. The uncertainty weight type reduction function is uncertainty conform.

Proof. Follows from Theorems 2 and 10.

Theorem 12. The uncertainty weight type reduction function ignores indifference.

Proof.

$$
f_{\mathrm{UW}}(0,1)=\frac{1}{2}(0+1) \cdot(1+0-1)^{\alpha}=0
$$

Fig. 4 shows the graphs of the UW function for $\alpha \in\{0.2,1,5\}$. All three graphs are nonlinear but smooth, and contain the points $(\underline{u}, \bar{u}, u)=(0,0,0),(1,1,1)$, and $(0,1,0)$, corresponding to ignorance of indifference. For $\alpha=0.2$ (left), the UW function looks very similar to the NT function at Fig. 3, only the very edge at $\underline{u}=0$ and $\bar{u}=1$ is bent from $u=0.5$ to $u=0$ (ignorance of indifference). Depending on the value of the parameter $\alpha$ the graph of the UW function is bent in different ways. It is monotonically increasing along the $\underline{u}$ axis with increasing $(\alpha=0.2)$ or decreasing slope $(\alpha=5)$. It is however not generally monotonic along the $\bar{u}$ axis. Consider for example the front edge curve at $\underline{u}=0$ which is distinctly bent up for medium values of $\bar{u} \approx 0.5$ for $\alpha=0.2$ (left) and $\alpha=1$ (center). For $\alpha=5$ (right), the UW function always yields very small memberships $u \approx 0$ except near the main diagonal at $\underline{u}=\bar{u}$, where it yields $u=\underline{u}=\bar{u}$ (type -1 consistency). For $\alpha=1$ (center), the UW function is quite flat along the $\bar{u}$ axis, and almost linearly increases along the $\underline{u}$ axis, so it approximates the function $u=\underline{u}$.

\section{Linear Type Reduction}

Besides NT and UW we can construct infinitely many type reduction functions $f$. For reasons of simplicity we first consider linear functions and derive specific instances of linear functions trying to satisfy our five proposed properties. So we start with the general case of linear functions for type reduction

$$
u=a \cdot \underline{u}+b \cdot \bar{u}+c
$$



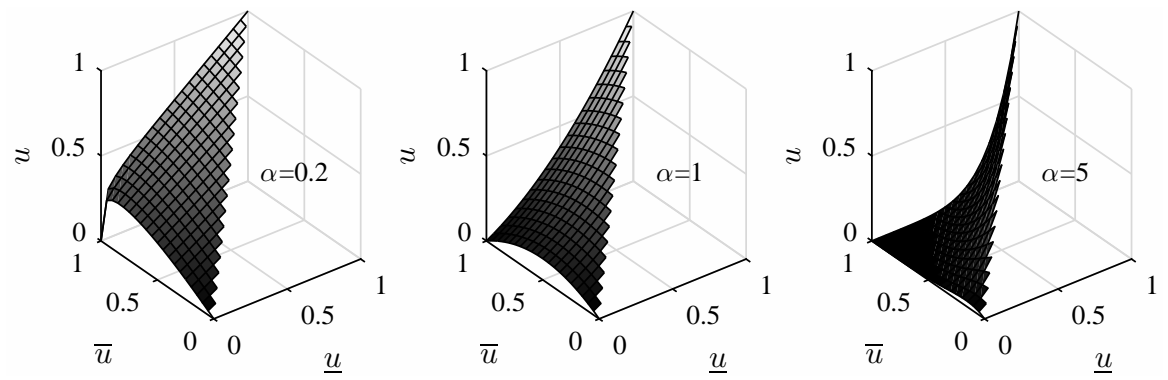

Figure 4: Graphs of the UW function for $\alpha \in\{0.2,1,5\}$.

with arbitrary parameters $a, b, c \in \mathbb{R}$. If we require type- 1 consistency, then we obtain

$$
\begin{aligned}
& \underline{u}=0, \quad \bar{u}=0, \quad u=0 \quad \Rightarrow \quad c=0 \\
& \underline{u}=1, \quad \bar{u}=1, \quad u=1 \quad \Rightarrow \quad 1=a+b+c \quad \Rightarrow b=1-a
\end{aligned}
$$

and so

$$
u=a \cdot \underline{u}+(1-a) \cdot \bar{u}
$$

If we further require type -2 consistency, then we obtain

$$
\begin{aligned}
& \underline{u} \leq a \cdot \underline{u}+(1-a) \cdot \bar{u} \quad \Rightarrow \quad(1-a) \cdot(\bar{u}-\underline{u}) \geq 0 \quad \Rightarrow \quad a \leq 1 \\
& a \cdot \underline{u}+(1-a) \cdot \bar{u} \leq \bar{u} \quad \Rightarrow \quad a \cdot(\bar{u}-\underline{u}) \geq 0 \quad \Rightarrow \quad a \geq 0
\end{aligned}
$$

which leads us to

Definition 6. The consistent linear type reduction (CLTR) function is defined as

$$
f_{\text {CLTR }}(\underline{u}, \bar{u})=a \cdot \underline{u}+(1-a) \cdot \bar{u}
$$

with $a \in[0,1]$

While the Nie-Tan type reduction function computes the plain average of the upper and lower membership grades, the CLTR function uses a convex combination of the upper and lower membership grades, which represents a weighted average with the weight $a$ for the lower membership grades and the weight $1-a$ 
for the upper membership grades. For $a=0$, CLTR will yield the upper membership function; for $a=0.5$, CLTR will yield the average of the upper and lower membership functions, which is also the result of the NT type reduction function (so the NT type reduction function is a special case of the CLTR function); and for $a=1$, CLTR will yield the lower membership function. For all other values of $a \in[0,1]$, CLTR will linearly interpolate between these special cases.

Theorem 13. The consistent linear type reduction function is type-1 and type2 consistent.

Proof. Follows immediately from the requirements for consistent linear type reduction functions (45)-(49).

Theorem 14. The consistent linear type reduction function is uncertainty conform if and only if $a \geq 0.5$.

Proof.

$$
\begin{gathered}
f_{\mathrm{CLTR}}\left(u^{*}-\Delta u, u^{*}+\Delta u\right) \\
=a \cdot\left(u^{*}-\Delta u\right)+(1-a) \cdot\left(u^{*}+\Delta u\right)=u^{*}+(1-2 a) \cdot \Delta u
\end{gathered}
$$

is monotocially decreasing with $\Delta u$ for $\alpha \in[0.5,1]$.

Theorem 15. The consistent linear type reduction function is strictly uncertainty conform if and only if $a>0.5$.

Proof. (51) is strictly monotocially decreasing with $\Delta u$ for $\alpha<(0.5,1]$.

Notice that the NT type reduction function is the only instance of the CLTR function that is uncertainty conform but not strictly uncertainty conform.

Theorem 16. The consistent linear type reduction function ignores indifference if and only if $a=1$.

Proof.

$$
f_{\mathrm{CLTR}}(0,1)=a \cdot 0+(1-a) \cdot 1=1-a=0 \quad \Leftrightarrow a=1
$$


Fig. 5 shows the graphs of the CLTR function for $a \in\{0.5,0.75,1\}$. All three graphs are triangles through $(\underline{u}, \bar{u}, u)=(0,0,0)$ and $(1,1,1)$. The third edge of each triangle is at $(0,1,1-a)$. The intersection of all CLTR graphs is the unit main diagonal through $(0,0,0)$ and $(1,1,1)$ (type-1 consistency). All three graphs are linear with non-negative slopes along $\underline{u}$ and $\bar{u}$. The slope is zero along $\bar{u}$ for $a=1$. All these three CLTR instances are uncertainty conform, because $a \in[0.5,1]$. The left graph $(a=0.5)$ corresponds to the NT function at Fig. 3. The right graph $(a=1)$ corresponds to the only CLTR function that ignores indifference, $u=\underline{u}$, which is approximately equal to the UW function for $\alpha=1$ (Fig. 4 center). For $a=1$, the CLTR function always yields $u=\underline{u}$, so the complete information about $\bar{u}$ is lost in this case. Hence, if we want to keep the information about both $\underline{u}$ and $\bar{u}$, and at the same type satisfy all our five properties, then we have to use a nonlinear type reduction function.
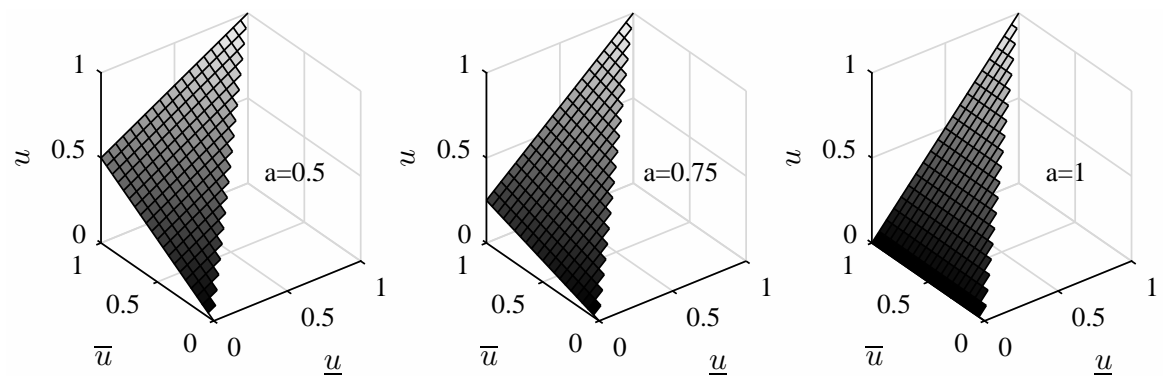

Figure 5: Graphs of the CLTR function for $a \in\{0.5,0.75,1\}$.

\section{Nonlinear Type Reduction}

As shown in the previous section, linear functions for type reduction may satisfy type -1 consistency, type -2 consistency, and (strict) uncertainty conformity, but we are not able to define any non-trivial $(a \neq 1)$ linear function for type reduction that ignores indifference. So, this property can only be satisfied by nonlinear type reduction functions. In this section we will therefore try to derive a nonlinear type reduction function that is as simple as possible and 
should be able to satisfy all five proposed properties.

To find such a function we start with the linear approach (44) and add a nonlinear term. This nonlinear term should be suitable to indicate indifference, so it should contain $\underline{u}$ and $\bar{u}$. In order to stay type -1 consistent, the nonlinear term should be zero when $\underline{u}=\bar{u}$. So, the nonlinear term could be defined as $g(\bar{u}-\underline{u})$ with a suitable function $g$ where $g(0)=0$. Here for simplicity we use a quadratic function for $g$ and obtain

$$
u=a \cdot \underline{u}+b \cdot \bar{u}+c+d \cdot(\bar{u}-\underline{u})^{2}
$$

with arbitrary parameters $a, b, c, d \in \mathbb{R}$. If we require type- 1 consistency, then we obtain just as in (45) and (46)

$$
\begin{aligned}
& \underline{u}=0, \quad \bar{u}=0, \quad u=0 \quad \Rightarrow \quad c=0 \\
& \underline{u}=1, \quad \bar{u}=1, \quad u=1 \quad \Rightarrow \quad 1=a+b+c \quad \Rightarrow b=1-a
\end{aligned}
$$

and so

$$
u=a \cdot \underline{u}+(1-a) \cdot \bar{u}+d \cdot(\bar{u}-\underline{u})^{2}
$$

If we further want to ignore indifference, then we obtain

$$
\underline{u}=0, \quad \bar{u}=1, \quad u=0 \quad \Rightarrow \quad 0=1-a+d \quad \Rightarrow \quad d=-(1-a)
$$

and so

$$
u=a \cdot \underline{u}+(1-a) \cdot \bar{u}-(1-a) \cdot(\bar{u}-\underline{u})^{2}
$$

If we further require type- 2 consistency, then we obtain

$$
\begin{aligned}
& \underline{u} \leq a \cdot \underline{u}+(1-a) \cdot \bar{u}-(1-a) \cdot(\bar{u}-\underline{u})^{2} \\
& \quad \Rightarrow \quad(1-a) \cdot(\bar{u}-\underline{u}) \geq(1-a) \cdot(\bar{u}-\underline{u})^{2} \quad \Rightarrow \quad a \leq 1
\end{aligned}
$$

and

$$
\begin{aligned}
& a \cdot \underline{u}+(1-a) \cdot \bar{u}-(1-a) \cdot(\bar{u}-\underline{u})^{2} \leq \bar{u} \\
& \quad \Rightarrow \quad a \cdot(\bar{u}-\underline{u}) \geq(a-1) \cdot(\bar{u}-\underline{u})^{2} \quad \Rightarrow \quad a \geq 0
\end{aligned}
$$

which leads us to 
Definition 7. The consistent quadratic type reduction (CQTR) function is defined as

$$
f_{\mathrm{CQTR}}(\underline{u}, \bar{u})=a \cdot \underline{u}+(1-a) \cdot \bar{u}-(1-a) \cdot(\bar{u}-\underline{u})^{2}
$$

with $a \in[0,1]$

The CQTR function (61) is equal to the CLTR function (50) minus the term $(1-a) \cdot(\bar{u}-\underline{u})^{2}$ which is zero for minimum uncertainty $(\underline{u}(x)=\bar{u}(x))$ and maximal (equal to $1-a$ ) for maximum uncertainty $(\underline{u}(x)=0, \bar{u}(x)=1$ ), and which increases quadratically with the uncertainty. For $a=0$, CQTR will yield the upper membership function minus the quadratic uncertainty, $\bar{u}-(\bar{u}-\underline{u})^{2}$; and for $a=1$, CQTR will simply yield the lower membership function $\underline{u}$. For all other values of $a \in[0,1]$, CQTR will linearly interpolate between these two cases.

Theorem 17. The consistent quadratic type reduction function is type-1 and type-2 consistent, and ignores indifference.

Proof. Follows immediately from the requirements for consistent linear type reduction functions (54)-(60).

Theorem 18. The consistent quadratic type reduction function is uncertainty conform if and only if $a \geq 0.5$.

Proof.

$$
\begin{gathered}
f_{\mathrm{CQTR}}\left(u^{*}-\Delta u, u^{*}+\Delta u\right) \\
=a \cdot\left(u^{*}-\Delta u\right)+(1-a) \cdot\left(u^{*}+\Delta u\right)-(1-a) \cdot(2 \Delta u)^{2} \\
=u^{*}+(1-2 a) \cdot \Delta u-4(1-a) \Delta u^{2}
\end{gathered}
$$

is monotocially decreasing with $\Delta u$ for $a=1$. For $a<1$ consider the slope

$$
\frac{\partial f_{\mathrm{CQTR}}}{\partial \Delta u}=1-2 a-8(1-a) \Delta u
$$

This slope is negative or zero for any $a<1$ if $1-2 a \leq 0 \Rightarrow a \in[0.5,1)$. So, $a \in[0.5,1]$. 
Theorem 19. The consistent linear type reduction function is strictly uncertainty conform if and only if $a>0.5$.

Proof. (62) is strictly monotocially decreasing with $\Delta u$ for $a=1$. (63) is negative for any $a<1$ if $1-2 a<0 \Rightarrow a \in(0.5,1)$. So, $a \in(0.5,1]$.

Fig. 6 shows the graphs of the CQTR function for $a \in\{0.5,0.75,1\}$. All three graphs contain the points $(\underline{u}, \bar{u}, u)=(0,0,0),(1,1,1)$, and $(0,1,0)$ (ignorance of indifference). All three graphs are strictly monotonically increasing along $\underline{u}$ with non-increasing slopes. For $a=1$ (right), the graph is linear; here the CQTR function is equal to the CLTR function for $a=1$ (Fig. 5 right), $u=\underline{u}$. This is the only intersection point of the CLTR and CQTR function instances. For $a=0.5$ (left) and $a=0.75$ (center), the graph of the CQTR function looks somewhat similar to the graph of the UW function for $\alpha=1$ (Fig. 4 center) but the front edge curve is bent more strongly.
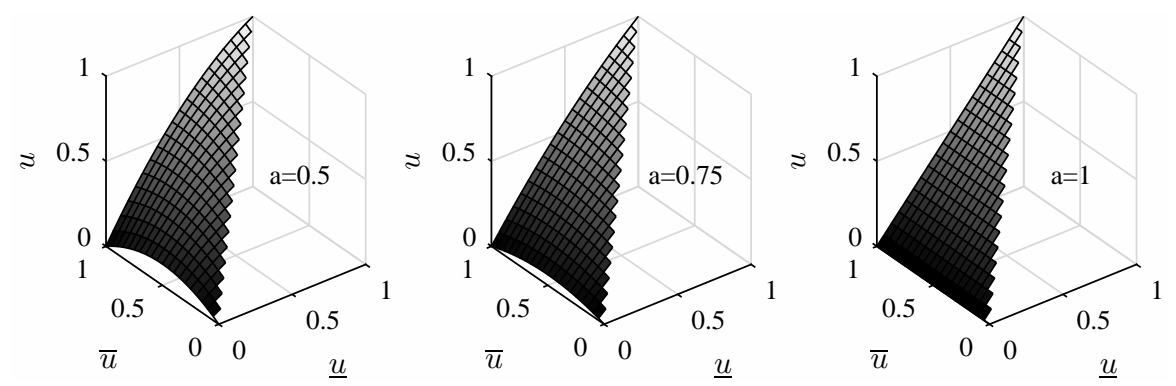

Figure 6: Graphs of the CQTR function for $a \in\{0.5,0.75,1\}$.

\section{Summary and Examples}

The properties of the NT, UW, CLTR, and CQTR functions are summarized in Table 1. The NT function is not strictly uncertainty conform and does not ignore indifference. The UW function is not type-2 consistent. CLTR is uncertainty conform for $a \in[0.5,1]$ and strictly uncertainty conform for $a \in$ $(0.5,1]$ but ignores indifference only for $a=1$. If we ignore this trivial special 
case, the only function considered here that satisfies all five properties is CQTR for $a \in(0.5,1]$.

Table 1: Properties of the NT, UW, CLTR, and CQTR functions.

\begin{tabular}{|l|c|c|c|c|}
\cline { 2 - 5 } \multicolumn{1}{c|}{} & NT & UW & CLTR & CQTR \\
\hline type-1 consistent & $\bullet$ & $\bullet$ & $\bullet$ & $\bullet$ \\
\hline type-2 consistent & $\bullet$ & & $\bullet$ & $\bullet$ \\
\hline uncertainty conform & $\bullet$ & $\bullet$ & & \\
\hline strictly uncertainty conform & & $\bullet$ & & \\
\hline ignores indifference & & $\bullet$ & & $\bullet$ \\
\hline
\end{tabular}

only for $a \in(0.5,1]$ or $[0.5,1], \bigcirc$ only for $a=1$

To illustrate the practical relevance of these properties we look at three selected interval type-2 fuzzy sets and present the results for the four different (families of) type reduction functions.

Fig. 7 left illustrates type -2 consistency for the interval type -2 membership function (shaded area) computed as

$$
\begin{aligned}
& \underline{u}(x)=1.8 \cdot((x-0.3) \cdot(x-0.5) \cdot(x-0.7)+0.15) \\
& \bar{u}(x)=3.5 \cdot((x-0.2) \cdot(x-0.6) \cdot(x-1)+0.25)
\end{aligned}
$$

These polynomial functions represent an example for smooth and continuous membership functions where the individual options possess different levels of preference and also different levels of uncertainty, where preference and uncertainty are not directly correlated. The maximum of the upper membership is at $x \approx 0.3$, but here the uncertainty is quite high. For $x \approx 1$ the upper membership is quite high but the uncertainty is much lower, so this area may be considered as a preferable choice in defuzzification. For this example NT (solid), CLTR ( $a=0.75$, dotted $)$, and CQTR ( $a=0.75$, dash-doted) yield type-1 membership functions that pass through the shaded area between $\underline{u}(x)$ and $\bar{u}(x)$. Only UW ( $\alpha=1$, dashed) yields a type -1 membership function that partially lies outside 
the shaded area. As illustrated in this example, NT, CLTR, and CQTR are type -2 consistent and therefore yield memberships inside the uncertainty range of the type-2 memberships (between the lower and upper type-2 membership), whereas UW is not type- 2 consistent and therefore may yield memberships outside this uncertainty range.
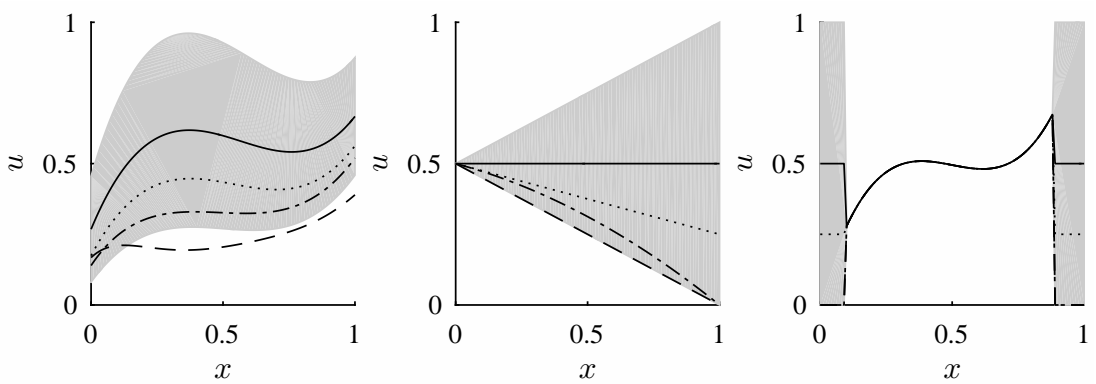

Figure 7: Example applications of type reduction functions: NT (solid), UW ( $\alpha=1$, dashed), CLTR $(a=0.75$, dotted), CQTR ( $a=0.75$, dash-doted).

Fig. 7 center illustrates strict uncertainty conformity for the interval type-2 membership function

$$
\begin{aligned}
& \underline{u}(x)=0.5 \cdot(1-x) \\
& \bar{u}(x)=0.5 \cdot(1+x)
\end{aligned}
$$

This is an example where the average degree of preference is constant but where the uncertainty increases linearly with $x$, from zero at $x=0$ to maximum uncertainty at $x=1$, so we may prefer the option with minimum uncertainty in defuzzification. For this example UW ( $\alpha=1$, dashed), CLTR ( $a=0.75$, dotted), and CQTR ( $a=0.75$, dash-doted) take into account the uncertainty and yield lower type-1 membership values for increasing uncertainty (increasing $x$ ). Only NT (solid) ignores the different levels of uncertainty and always yields a constant type-1 membership value of 0.5 . As illustrated in this example, UW, CLTR, and CQTR are (for $a \in(0.5,1]$ ) strictly uncertainty conform and therefore yield lower type- 1 membership when the uncertainty is high, whereas 
$\mathrm{NT}$ is not strictly uncertainty conform and therefore does not recognize different levels of uncertainty.

Fig. 7 right illustrates ignorance of indifference for the interval type-2 membership function

$$
\begin{aligned}
& \underline{u}(x)= \begin{cases}4.5 \cdot((x-0.3) \cdot(x-0.5) \cdot(x-0.7)+0.11) & \text { for } x \in[0.1,0.9] \\
0 & \text { otherwise }\end{cases} \\
& \bar{u}(x)= \begin{cases}4.5 \cdot((x-0.3) \cdot(x-0.5) \cdot(x-0.7)+0.11) & \text { for } x \in[0.1,0.9] \\
1 & \text { otherwise }\end{cases}
\end{aligned}
$$

This is an example where the uncertainty is zero in a certain interval (here $x \in[0.1,0.9])$, so here the memberships are of type- 1 , and outside this interval we have maximum uncertainty, $\underline{u}(x)=0$ and $\bar{u}(x)=1$, for $x \in[0,0.1)$ and for $x \in(0.9,1]$ (shaded areas), so we may want to ignore the areas of extreme uncertainty in defuzzification. For this example all four methods correctly recognize the type -1 part of this membership function. However, only UW ( $\alpha=1$, dashed) and CQTR ( $a=0.75$, dash-doted) yield zero type-1 membership values for the two areas of indifference, whereas NT (solid) yields 0.5, and CLTR ( $a=0.75$, dotted) yields 0.25 there. As illustrated in this example, UW and CQTR (for $a \in(0.5,1])$ ignore indifference and therefore always yield zero membership for options with maximal uncertainty, whereas NT and CLTR (for $a \neq 1$ ) do not ignore indifference and therefore yield non-zero memberships here.

\section{Conclusions}

As a first step towards a theoretical foundation of type reduction we have introduced a set of five mathematical properties and illustrated some relations between these properties.

Our experimental studies have proven the merit of objective mathematical criteria for the study of type reduction operators. The Nie-Tan (NT) method is a good choice for type reduction when it is important to be type- 2 consistent, but not important to be strictly uncertainty conform or to ignore indifference. The uncertainty weight (UW) method is a good choice when it is important to be 
strictly uncertainty conform and to ignore indifference, but when not important to be type- 2 consistent.

Further we have proposed two new type reduction methods, consistent linear type reduction (CLTR) and consistent quadratic type reduction (CQTR). CLTR satisfies all our five properties except ignorance of indifference, and CQTR completely satisfies all five properties (for $a \in(0.5,1]$ ). So, CLTR and CQTR are considered good alternatives to NT and UW with attractive mathematical properties.

The purpose of this paper is to provide a foundation of the theory of type reduction. Given the width of this field, this paper has to leave many important aspects open for further research, for example: Can we find a real-world application example where satisfying our five properties will improve system performance? How can our list of five properties of type reduction be reasonably refined and/or extended? How can similar properties be defined for other types of (non-interval) type-2 fuzzy sets? How does type reduction relate to averaging and ignorance functions [5]? How can we define properties of type reduction for non-pointwise type reduction, for example in constrained fuzzy sets [8], where a type -2 fuzzy set is generated by wobbling a type -1 fuzzy set along the horizontal axis? What kind of properties can we realize with nonlinear type reduction functions other than UW and CQTR? Where can type reduction be applied beyond defuzzification?

\section{References}

[1] E. Barrenechea, H. Bustince, M. Pagola, J. Fernández, Construction of interval-valued fuzzy entropy invariant by translations and scalings, Soft Computing 14(9), 2010, pp. 945-952.

[2] G. Beliakov, H. Bustince, T. Calvo, A Practical Guide to Averaging Functions, Springer, 2016

[3] H. Bustince, J. Fernandez, H. Hagras, F. Herrera, M. Pagola, E. Barrenechea, Interval type-2 fuzzy sets are generalization of interval-valued 
fuzzy sets: Toward a wider view on their relationship, IEEE Transactions on Fuzzy Systems 23 (5) (2015) 1876-1882.

[4] H. Bustince, J. Fernández, A. Kolesárová, R. Mesiar, Directional monotonicity of fusion functions, European Journal of Operational Research 244(1), 2015, pp. 300-308.

[5] H. Bustince, M. Pagola, E. Barrenechea, J. Fernández, P. Melo-Pinto, P. Couto, H. R. Tizhoosh, and J. Montero, Ignorance functions. An application to the calculation of the threshold in prostate ultrasound images, Fuzzy sets and Systems 161(1), 2010, pp. 20-36.

[6] C. Chen, R. John, J. Twycross, J. Garibaldi, A direct approach for determining the switch points in the Karnik-Mendel algorithm, IEEE Transactions on Fuzzy Systems.

[7] D. Driankov, H. Hellendoorn, M. Reinfrank, An Introduction to Fuzzy Control, Springer, Berlin, 1995.

[8] J. M. Garibaldi, S. Guadarrama, Constrained type-2 fuzzy sets, in: IEEE Symposium on Advances in Type-2 Fuzzy Logic Systems, 2011, pp. 66-73.

[9] M. Gehrke, C. Walker, E. Walker, Some comments on interval valued fuzzy sets, International Journal of Intelligent Systems 11 (10) (1996) 751-759.

[10] M. B. Gorzałczany, A method of inference in approximate reasoning based on interval-valued fuzzy sets, Fuzzy Sets and Systems 21 (1) (1987) 1-17.

[11] N. N. Karnik, J. M. Mendel, Centroid of a type-2 fuzzy set, Information Sciences 132 (2001) 195-220.

[12] J. Li, R. John, S. Coupland, G. Kendall, On Nie-Tan operator and typereduction of interval type-2 fuzzy sets, IEEE Transactions on Fuzzy Systems.

[13] Q. Liang, J. M. Mendel, Interval type-2 fuzzy logic systems: Theory and design, IEEE Transactions on Fuzzy Systems 8 (5) (2000) 535-550. 
[14] E. H. Mamdani, S. Assilian, An experiment in linguistic synthesis with a fuzzy logic controller, International Journal of Man-Machine Studies 7 (1) (1975) 1-13.

[15] J. M. Mendel, R. I. John, F. Liu, Interval type-2 fuzzy logic systems made simple, IEEE Transactions on Fuzzy Systems 14 (6) (2006) 808-821.

[16] J. M. Mendel, M. R. Rajati, P. Sussner, On clarifying some definitions and notations used for type- 2 fuzzy sets as well as some recommended changes, Information Sciences 340-341 (2016) 337-345.

[17] M. Nie, W. W. Tan, Towards an efficient type-reduction method for interval type-2 fuzzy logic systems, in: IEEE International Conference on Fuzzy Systems, Hong Kong, 2008, pp. 1425-1432.

[18] W. Pedrycz, Fuzzy Control and Fuzzy Systems, 2nd Edition, Wiley, New York, 1993.

[19] S. Roychowdhury, W. Pedrycz, A survey of defuzzification strategies, International Journal of Intelligent Systems 16 (6) (2001) 679-695.

[20] T. A. Runkler, Selection of appropriate defuzzification methods using application specific properties, IEEE Transactions on Fuzzy Systems 5 (1) (1997) 72-79.

[21] T. A. Runkler, S. Coupland, R. John, Interval type-2 fuzzy decision making, International Journal of Approximate Reasoning 80 (2017) 217-224.

[22] T. A. Runkler, S. Coupland, R. John, Properties of interval type-2 defuzzification operators, in: IEEE International Conference on Fuzzy Systems, Istanbul, Turkey, 2015.

[23] T. A. Runkler, S. Coupland, R. John, C. Chen, Interval type-2 defuzzification using uncertainty weights, in: S. Mostaghim, C. Borgelt, A. Nürnberger (Eds.), Frontiers in Computational Intelligence, Springer, 2017. 
[24] T. A. Runkler, M. Glesner, A set of axioms for defuzzification strategies towards a theory of rational defuzzification operators, in: IEEE International Conference on Fuzzy Systems, San Francisco, 1993, pp. 1161-1166.

[25] J. J. Saade, H. B. Diab, Defuzzification techniques for fuzzy controllers, IEEE Transactions on Systems, Man, and Cybernetics, Part B 30 (1) (2000) 223-229.

[26] T. Takagi, M. Sugeno, Fuzzy identification of systems and its application to modeling and control, IEEE Transactions on Systems, Man, and Cybernetics 15 (1) (1985) 116-132.

[27] D. Wu, J. M. Mendel, Enhanced Karnik-Mendel algorithms, IEEE Transactions on Fuzzy Systems 17 (4) (2009) 923-934.

[28] D. Wu, M. Nie, Comparison and practical implementation of typereduction algorithms for type-2 fuzzy sets and systems, in: IEEE International Conference on Fuzzy Systems, 2011, pp. 2131-2138.

[29] L. A. Zadeh, Fuzzy sets, Information and Control 8 (1965) 338-353.

[30] L. A. Zadeh, The concept of a linguistic variable and its application to approximate reasoning, Information Sciences 8 (1975) 199-249, 9:43-80. 